Cuadernos de Trabajo Social

ISSN: 1988-8295

https://dx.doi.org/10.5209/cuts.79414

\title{
Los arrecifes de coral: una propuesta de revista sostenible para la comunidad de trabajo social
}

\author{
Maribel Martín Estalayo
}

Tomar el testigo de una revista como Cuadernos de Trabajo Social, que desde 1987 ha sido una leal y comprometida compañera en la consolidación de la disciplina de Trabajo Social, es un gesto de confianza de la comunidad universitaria a la que pertenezco y una responsabilidad personal. Por ello, antes de presentar el proyecto de revista en la Junta de Facultad para el periodo 2021-2025, realicé un ejercicio retrospectivo por los treinta y cuatro volúmenes que componían su larga vida. Disfrute, admiración y respeto me suscitaron las muchas compañeras y los compañeros de esta Facultad (antigua Escuela) que se remangaron en su rol de dirección, de secretaría, de autoría, de gestión, de evaluación o de asesoría. Casi podemos afirmar que toda la casa Complutense y las personas referentes que constituyen el territorio nacional, están representadas a lo largo y ancho de sus páginas. Emociona releer las cartas o editoriales, porque en todas ellas se desprende el ímpetu, el esfuerzo y el compromiso con la disciplina y la práctica social.

Y a la relectura se suma la suerte de la experiencia. Con Teresa Zamanillo, aprendí la importancia de escribir en trabajo social y el cuidado que merecen los espacios de reflexión e intercambio. Con Luis Nogués, pude comprender que todo proyecto es ideológico y tiene que estar al servicio de la transformación social. Y con Teresa G. Giráldez, me quedó muy claro que todo lo anterior no es posible si no se hace con dedicación, esfuerzo y una especie de optimismo estructural. Estas tres direcciones de revista están hoy muy presentes, por lo que nuestro proyecto representa continuidad y sintonía intelectual con quienes nos precedieron. Y, a su vez, es innegable que toda nueva etapa y las diferentes trayectorias personales y profesionales traen consigo un estilo propio y una estrenada declaración de intenciones.

Ahora bien, aún con todo el aprendizaje y la confianza institucional jamás hubiera encontrado el valor para coordinar este proyecto si no contara con un equipo de altura en el conocimiento, la comunicación y el compromiso con el trabajo social. Me acompañan en esta aventura Tuca Muriel (secretaria de redacción), Marta Blanco (revisora editorial de textos internacionales), Oscar Cebolla (revisor editorial de reseñas), Nidia José (becaria de apoyo a la revista) y un comité editorial y asesor bien dotado de personas referentes a nivel nacional e internacional en el ámbito de conocimiento del trabajo social y las ciencias sociales.

Este equipo entiende la revista como un espacio de encuentro y compromiso con la disciplina que nos reúne. Y, en este sentido, es una bonita coincidencia iniciar andadura a sus 35 años de vida. Quien conoce o aún celebra los compromisos, identificará que a esas alturas se festejan las bodas de coral. Dicen que los arrecifes de coral rebosan vida, pues ocupando menos del 1\% de la superficie oceánica, acogen a casi el $25 \%$ de las criaturas marinas. Se caracterizan por vivir en comunidad para su subsistencia y sus propiedades mágicas nos protegen del peligro y la enfermedad. Como tantos otros elementos del planeta, su existencia está amenazada por factores humanos y se prevé que desaparezca una parte importante de estos arrecifes en los próximos años. Este símil nos anima a poner en el centro del proyecto de revista una idea y un deseo sustancial: este es un proyecto de todas y todos, un espacio universitario y profesional que, en medio de una pandemia mundial y una sociedad inscrita en un modelo neoliberal que empuja hacia el individualismo desenfrenado, nos pueda permitir reconocernos en una comunidad en la que vivir del modo más saludable y sostenible posible. Ojalá esta revista, cual arrecife de coral, rebose vida, acoja a todas las criaturas del mundo del trabajo social y, paradójicamente, prospere a pesar de estar rodeada de aguas oceánicas poco nutrientes.

Y a partir de esta idea y deseo, se desprende la siguiente declaración de intenciones y estilo propio:

- No vamos a resolver la tensión entre la academia y la profesión, la teoría y la práctica, la reflexión y la calle. Pero la capacidad de argumentación científica ha de ser una premisa fundamental. Si la universidad precisa reflexionar sobre lo que ocurre realmente y no desde la experiencia idealizada, la profesión también requiere de una interpretación rigurosa de sus prácticas que le permita avanzar. Establecer puentes y buscar equilibrios ha de favorecer la vocación científica de una revista al servicio de la disciplina y de la profesión. 
- Entendemos que alcanzar todos los grados académicos en la academia -especialmente con el Máster Oficial y el Doctorado- debe tener su correspondencia en la calidad y validez de los trabajos de investigación en el área de conocimiento de trabajo social y servicios sociales. Por tanto, la invitación y la potenciación de la investigación novel será una de nuestras prioridades. Y esto alude directamente a la convicción de una política editorial que priorice las aportaciones del área disciplinar.

- Queremos desarrollar y afianzar un plan de comunicación y difusión que permita dar una mayor visibilidad a los contenidos publicados, y que conecte con ese público más joven que usa las redes sociales a diario. Porque actualmente, en algunas bases de datos, las redes sociales han pasado a ser métricas tomadas en cuenta a la hora de medir el impacto de las publicaciones científicas. Y en esta línea de difundir y traspasar fronteras, animaremos a los y las articulistas a la internacionalización, esto es, invitarles a publicar en castellano y en inglés.

- Trabajaremos para mantener y aumentar los indicadores de impacto de las bases de datos y plataformas de evaluación en los que la revista ya está indexada. Asimismo, pretendemos analizar la revista en relación con las modificaciones sugeridas que hay que implementar gradualmente para su evaluación e incorporación en nuevas bases de datos. Este objetivo pasa a regir parte de nuestra estrategia, puesto que la existencia y la valoración forman parte indisociable de toda dinámica del reconocimiento. Pero también somos conscientes, por una parte, de la dificultad que supone compatibilizar los estándares exigidos por la comunidad científica internacional con los lineamientos éticos y de identidad de CTS; y, por otra parte, por la dificultad de compatibilizar dichos estándares con la realidad de las revistas científicas españolas, principalmente las que pertenecen al área de Ciencias Sociales.

- Y, por último, nuestro compromiso con la sostenibilidad, el acceso libre y el traspaso de fronteras, hace que este número 35 (1) se estrene enteramente como revista digital. Veníamos de un tiempo de transición donde convivía el soporte y las formas analógicas con la inmersión en los entornos digitales. Hemos querido dar el salto definitivo para poder pensar y hacer en digital, esto es: centrarnos en la mejora y la visualización de los contenidos, el perfeccionamiento de los procesos editoriales y la periodicidad, la posibilidad de aumentar la extensión de los artículos, la utilización del presupuesto para la difusión y la internacionalización, etcétera.

En resumen, esta nueva etapa y nuevo equipo quiere ponerse al servicio del aumento de conocimiento de la disciplina de Trabajo Social, del estímulo en la formación del estudiantado de Grado, Máster y Doctorado, del intercambio de reflexiones, debates y avances en el ámbito nacional e internacional, en la profesión y en la universidad, y de una Facultad de Trabajo Social de la Universidad Complutense de Madrid en proceso de crecimiento que se ofrece, como tantas otras veces, como espacio de todas y todos. Pues como sugeríamos en las anteriores líneas con el símil de los arrecifes de coral, es nuestra manera de convivir, contribuir y mitigar los envites del individualismo para hacer de nuestro espacio académico un lugar cada día más sostenible.

Y ya dispuestas en la tarea, presentamos los ocho artículos que conforman el primer número del volumen 35 de la revista, de los cuales uno de ellos es en inglés y dos de ellos se ofrecen en ambas versiones -castellano e inglés-. Los temas sobre los que nos invitan a reflexionar dan cuenta de la diversidad y el abanico de inquietudes que están presentes en toda intervención social: envejecimiento activo, diversidad sexual, calidad en servicios sociales, ética, trabajo social sanitario, recursos para personas sin hogar e inseguridad alimentaria, familias víctimas de desaparición.

El primer artículo publicado en inglés y bajo el título Promoting mental fitness in the context of active ageing policies. Comparative analysis of action programme objectives in eight countries, es un estudio comparativo de las iniciativas de ocho países europeos en torno a los programas de aptitud mental para el mantenimiento del bienestar emocional y psicológico de los adultos mayores. Carmen Romo Parra, Miguel Ángel García-Martín, María Teresa Vera Balanza, José Jesús Delgado Peña, indagan mediante un análisis de contenido cualitativo en 116 programas y servicios para examinar los objetivos planteados en términos de actitudes, conocimientos y capacidades. La información derivada de dicho análisis proporciona elementos a tener en cuenta en las políticas de planificación del contexto de envejecimiento de la población europea.

El segundo artículo viene de la mano del autor Jair Eduardo Restrepo Pineda. Percepciones, imaginarios y conocimientos sobre diversidad sexual entre estudiantes de trabajo social, nos permite conocer los presupuestos que el estudiantado de trabajo social en Colombia trae consigo con relación a los temas de diversidad sexual e identidad de género. Analizar dichos presupuestos y trabajar la posible conformación o mantenimiento de prejuicios y estereotipos sobre esta población, es una labor indispensable para que los y las futuras profesionales actúen desde el respeto y el reconocimiento de la diversidad sexual.

Ma Victoria Ochando Ramírez, con su artículo Aplicación del Modelo EFQM 2020 para Servicios Sociales, establece un diálogo entre sistema y modelo de calidad. En ese intercambio se identifican los criterios y los valores que los sostienen y se investiga sobre las limitaciones y los puntos en común que los pueden hacer complementarios. La autora parte de una clara premisa: la aplicación de la calidad en servicios sociales ha de garantizar el respeto de su singularidad, esto es, la adaptación al entorno y la priorización de la dignidad de las personas usuarias. 
Y recién celebrado el 200 aniversario del nacimiento de Concepción Arenal, su influencia y aporte sigue estando muy presente en los albores del trabajo social con el siguiente artículo de Francisco Idareta Goldaracena. En esta ocasión, podemos acceder en castellano y en inglés al texto De la ethica misericordis de Concepción Arenal a la ethica cordis de Adela Cortina: origen y actualidad de la ética del Trabajo Social en España. Razón y corazón orquesta el estudio de estas dos contribuciones sobre ética y justicia social y, a su vez, el autor nos descubre planteamientos y sintonías presentes entre la modernidad y la posmodernidad.

José Pablo Calleja Jiménez ilustra con su artículo El Trabajo Social Sanitario en la lucha contra La Covid-19. Las labores de rastreo de contactos y el refuerzo al sistema sanitario, la experiencia de los profesionales del trabajo social en Asturias y el lugar que ocupan en el control de enfermedades infecciosas. El autor justifica a lo largo de su estudio la importancia de la dimensión social en el diagnóstico clínico y el interés de un abordaje interdisciplinar que pueda atender en toda su complejidad situaciones pandémicas como la actual.

El artículo Análisis del conflicto en un recurso para personas sin hogar: diseño y validación de la hoja de registro de los factores asociados, pone sobre la mesa las situaciones de conflicto que se derivan de la institucionalización de un espacio de convivencia y atención residencial como pueden ser los centros de acogida de personas sin hogar. Para ello, José Manuel Díaz González; Juan Antonio Rodríguez Hernández; Manuel José Rosales Álamo, describen, construyen y validan una herramienta de análisis a partir de las hojas de registro que recogen diversas categorías sobre el conflicto en ese contexto. Los autores entienden el conflicto como un elemento consustancial a las relaciones humanas y, por ello, ponen el foco en la importancia de su atención y estudio por parte de la comunidad científica para ponerlo al servicio de la intervención social.

El séptimo artículo, Iniciativas para enfrentar el hambre y la inseguridad alimentaria en la Comunidad de Madrid: ¿está en crisis el modelo hegemónico de reparto de alimentos?, está escrito por Araceli Serrano Pascual; José Ramón González Parada y Marian Simón Rojo. A lo largo de su análisis, nutrido de la experiencia de un proyecto de aprendizaje colaborativo de la Universidad Complutense, el Observatorio para la Garantía del Derecho a la Alimentación y la Carta contra el Hambre durante varios años, se ponen de relieve las contradicciones y tensiones que presenta el modelo hegemónico de protección alimentaria de la Comunidad de Madrid y la respuesta de sus distintos intervinientes.

El octavo y último artículo, escrito por Luz Alejandra Escalera Silva, Sandra Rubí Amador Corral y María Luisa Castellanos López y titulado Atención social especializada a familias de personas víctimas de desaparición durante la contingencia Covid-19, pone el foco en una realidad singular del país mexicano y los efectos de la pandemia en la vulnerabilidad de las familias víctimas de esta situación. Se presenta el trabajo social espacio de coordinación interinstitucional imprescindible en dicha complejidad.

Esperamos que los distintos temas de este nuevo volumen de revista sean de su interés y estimule nuevas líneas de reflexión y práctica en el campo de lo social. 